\title{
Mechanism of Hard-Roof Rock Burst Control by the Deep-Hole Blasting: Numerical Study Based on Particle Flow
}

\author{
Anliang Lu $\mathbb{D}^{1},{ }^{1,2}$ Linming Dou $\mathbb{D}^{1,2}$ Jinzheng Bai $\mathbb{D}^{1,2}$ Yanjiang Chai, ${ }^{1,2}$ Kunyou Zhou, ${ }^{1,2}$ \\ Jiliang Kan, ${ }^{1,2}$ Jinrong Cao, ${ }^{1,2}$ and Shikang Song ${ }^{3}$ \\ ${ }^{1}$ Key Laboratory of Deep Coal Resource Mining, Ministry of Education, China University of Mining and Technology, \\ Xuzhou 221116, China \\ ${ }^{2}$ School of Mines, China University of Mining and Technology, Xuzhou 221116, China \\ ${ }^{3}$ Shaanxi Zhengtong Coal Industry Co., Ltd., Xianyang 713600, China
}

Correspondence should be addressed to Linming Dou; lmdou@126.com

Received 21 September 2021; Accepted 15 October 2021; Published 31 October 2021

Academic Editor: Fan Deyuan

Copyright (c) 2021 Anliang Lu et al. This is an open access article distributed under the Creative Commons Attribution License, which permits unrestricted use, distribution, and reproduction in any medium, provided the original work is properly cited.

In underground coal mines, the deep-hole blasting (DHB) technology is generally adopted for thick hard-roof control. This technology uses the energy released by explosives to weaken the energy storage capacity of hard roof so as to prevent hard-roof rock burst disasters. In this paper, a numerical simulation model of roof DHB was established based on particle flow and the damage range of single-hole blasting with concentrated cylindrical charge was studied. The temporal and spatial evolutions of overlying strata, the distribution of the force chain structure, and the working resistance of hydraulic pressure in the mining process before and after the application of DHB were contrastively analyzed. The following beneficial conclusions were drawn. The blasting-induced single-hole damage range is generally characterized by annular zoning. After the application of DHB, overall the collapse morphology of the key strata in the mining process changes from long-distance instantaneous slipping instability to stratified short-arm stepped synergistic subsidence. The density and strength of force chains in the overburden are notably reduced; the peak value of compressive force chain strength in the key strata in the mining process falls by $17.85 \%$ as a result of DHB. The monitoring results of the working resistance of hydraulic support reveal that the DHB technology can effectively shorten the step distance of periodic weighting and reduce the variation amplitude of overburden load during weighting. In summary, the mechanism of hard-roof rock burst control by DHB is reflected by both static load reduction and dynamic load reaction.

\section{Introduction}

Rock burst, characterized by the sudden and violent release of massive elastic strain energy accumulated in coal rock, poses a major threat to the health and safety of workers in coal mining [1-3]. According to the theory of dynamic load and static load superposition [4-6], when the static load and dynamic load in coal rock around the mining space overlap and exceed the critical load, rock burst will occur. Thick hard roof can control rock burst from two aspects. First, it can easily form hanging roof at the edge of the gob due to its good mechanical properties, which transfers the overlying load to the coal rock around the gob and leads to the formation of locally high-static-load stress concentration.
Second, when the length of the hanging roof reaches the ultimate collapse distance, the roof breaks and loses stability instantaneously, releasing strong dynamic load, which promotes the occurrence of rock burst [7] (Figure 1). In the hope of effectively controlling the hard-roof rock burst, active pressure relief measures are required to reduce the high static load and dynamic load around the mining space.

The key to prevention of hard-roof rock burst is the modification of thick hard roof by weakening the strata structure. At present, the commonly adopted methods include deep-hole blasting [9] and hydraulic fracturing [10]. The principle of DHB for lowering rock burst risk is to use the energy released by explosives to increase the beddings and fractures of specific strata, weaken their integrity and 


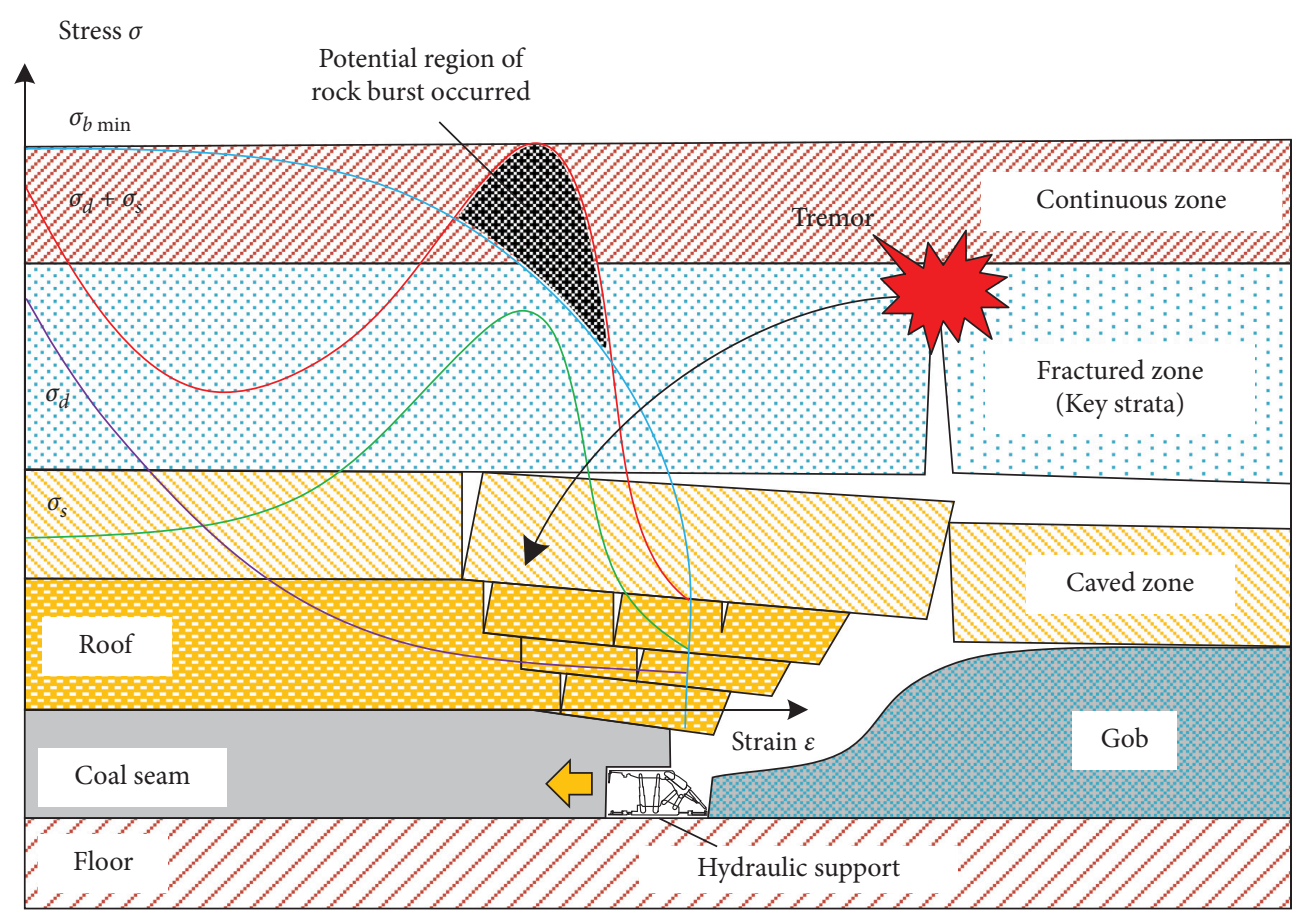

FIgURE 1: Schematic diagram of hard-roof rock bursts [8].

strength, and advance the breakage of hanging roof [11]. DHB boasts the following advantages. (1) The equipment is easy to operate and flexible to move, suitable for operations of underground narrow space. (2) The technology is mature with a convenient operation process; the basic procedure just requires three steps, that is, "drilling-charging-initiating." (3) It is generally feasible for dealing with thick hard roofs of various occurrence types. Therefore, DHB is considered as an effective and active pressure relief method for the control of hard-roof rock burst and has been widely adopted in mines with similar engineering conditions [12].

In recent decades, with the application of DHB technology on engineering sites, scholars have carried out fruitful researches on the technical principle and effect evaluation of roof blasting. Wang et al. [13] studied the rock stress field and crushing range under the action of high-energy explosive stress wave, optimized the parameters of deep-hole prefracturing blasting and revealed the blasting mechanism of controlled roof caving. Konicek et al. [14] proposed a seismic effect parameter for evaluating the stress release effect of roof blasting on overlying strata. Chen et al. [15] studied the crack evolution law under the synergistic action of dynamic and static loads in deep-hole presplitting blasting. Yang et al. [16] analyzed the crack shape after presplitting blasting and obtained the deformation characteristics and failure mechanism of roof deep-hole blasting. Banadaki et al. [17] studied the rock fracture mode induced by explosion stress wave through single-hole blasting experiment. Through theoretical calculation and numerical simulation, Meng et al. [18] proved that shaped charge blasting could achieve directional propagation of blasting cracks and relieve mining pressure. Gao et al. [19] believed that the directional blasting technology of roof affected the stress distribution and failure state around the roadway and protected the roof from breakage or destruction. Existing researches indicate that roof DHB can effectively change strata structure. However, there are few studies on the mechanism of DHB technology in controlling hard-roof rock burst, and the research conclusions fail to support the effect verification and parameter optimization of roof DHB in engineering sites.

In this study, a numerical simulation model of roof DHB was established through Particle Flow Code (PFC), and the damage range of single-hole blasting with concentrated cylindrical charge was studied. Moreover, the spatial and temporal evolutions of overlying strata, the distribution of the force chain structure, and the working resistance of hydraulic support in the two schemes (i.e., the nonblasting scheme and the blasting scheme) were contrastively analyzed. This study helps to reveal the mechanism of DHB technology in controlling hard-roof rock burst.

\section{Engineering Background}

2.1. Geological Conditions. Gaojiapu Coal Mine is located in Xianyang City, Shaanxi Province, China, with an annual output of 5 million tons. The selected 302 working face is located in the 403 panel. The specific parameters of the working face are as follows: the width of inclination is $190 \mathrm{~m}$; the strike length is about $1275 \mathrm{~m}$; the average inclination angle of $4 \#$ coal seam is $5^{\circ}$, and the average thickness of it is $8.9 \mathrm{~m}$. The fully mechanized longwall caving mining method is adopted for recovery, and the roof behind the hydraulic support can collapse directly.

The comprehensive histogram of strata is shown in Figure 2. The strata above $4 \#$ coal seam include $2.85 \mathrm{~m}$ of mudstone, $4.04 \mathrm{~m}$ of fine sandstone, $14.21 \mathrm{~m}$ of medium 


\begin{tabular}{|c|c|c|c|c|}
\hline & Thickness $(\mathrm{m})$ & Depth $(\mathrm{m})$ & Lithology & Remarks \\
\hline & 5.96 & 923.79 & Sandy mudstone & \\
\hline & 7.24 & 931.03 & Mudstone & \\
\hline & 2.66 & 933.69 & Siltstone & \\
\hline & 6.81 & 940.50 & Sandy mudstone & \\
\hline & 7.23 & 947.73 & Grit mudstone & \\
\hline & 14.21 & 961.94 & Medium mudstone & Key strata \\
\hline & 8.12 & 970.06 & Fine sandstone & \\
\hline & 4.04 & 974.10 & Siltstone & \\
\hline & 2.85 & 976.95 & Mudstone & Immediate roof \\
\hline & 8.90 & 985.85 & 4\# coal seam & \\
\hline & 1.98 & 987.83 & Carbon Mudstone & Immediate floor \\
\hline & 5.70 & 993.53 & Fine sandstone & \\
\hline & & & & \\
\hline & & & &
\end{tabular}

Figure 2: Comprehensive histogram of strata.

sandstone, and $7.23 \mathrm{~m}$ of coarse sandstone, while the strata below include $1.98 \mathrm{~m}$ of carbonaceous mudstone and $5.70 \mathrm{~m}$ of fine sandstone. According to the mechanical test results, the uniaxial compressive strength of medium sandstone is $118.72 \mathrm{MPa}$, and $4 \#$ coal seam is rather prone to rock burst. Medium sandstone, which is of high strength, high thickness, high integrity, and undeveloped joints, serves as the hard key strata of the 302 longwall working face, and is likely to form a large hanging roof during mining.

2.2. Process of Deep-Hole Blasting on Site. To avoid hard-roof rock burst induced by the sudden breakage of sandstone in the hard thick strata, roof DHB was carried out on the mining sides of the two roadways before the recovery of the 302 working face (Figure 3). The design parameters of the technology mainly include the following: blasting stratum, charge quantity, borehole inclination angle, borehole length, borehole diameter, borehole row spacing, and sealing length. They are determined based on theoretical analysis and onsite practice experience. The operation process and parameters are as follows:

(1) The mining sides of the two roadways ahead of the 302 working face were drilled. Each group contained two boreholes 1\# and 2\#. The borehole diameter $\phi_{1}$ was $85 \mathrm{~mm}$; the inclination angles $\theta_{1}$ and $\theta_{2}$ of the boreholes were $50^{\circ}$ and $38^{\circ}$, respectively. The full lengths $L_{1}$ and $L_{2}$ of the boreholes were $48 \mathrm{~m}$ and $60 \mathrm{~m}$. The spacing $D$ between boreholes in each group was $20 \mathrm{~m}$.

(2) The permissible emulsion explosive for secondary coal mine was selected, and the positive continuous decoupling method was adopted for charging. The diameter $\phi_{2}$ of the charge roll was $60 \mathrm{~mm}$, and the radial decoupling charging coefficient $K$ was about 1.4. The loading quantity per meter was about $2.8 \mathrm{~kg}$, and the single-hole loading quantities $Q_{1}$ and $Q_{2}$ were $50 \mathrm{~kg}$ and $65 \mathrm{~kg}$, respectively. The holes were sealed with yellow mud and the lengths $L_{3}$ and $L_{4}$ were $30 \mathrm{~m}$ and $37 \mathrm{~m}$, respectively.

(3) The detonating cord series, millisecond-delay detonator initiation, and short delay blasting were

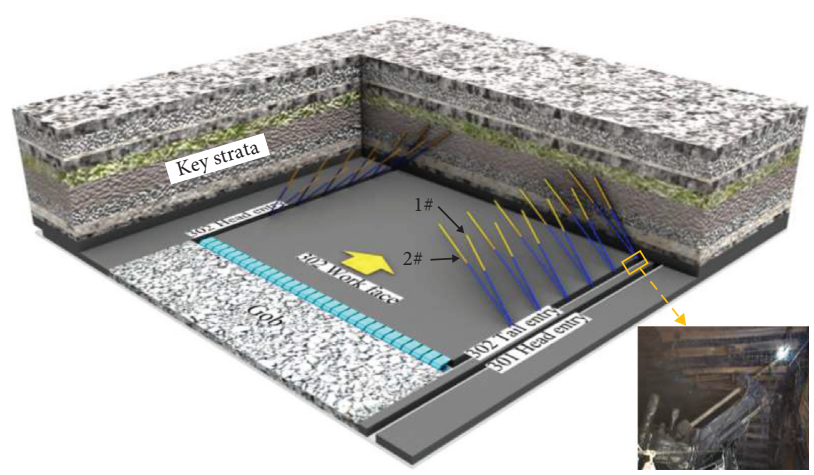

FIGURE 3: Schematic diagram of the operation process of roof deephole blasting.

adopted; two holes in each group were detonated at the same time.

\section{Numerical Simulation}

$\mathrm{PFC}^{2 \mathrm{D}}$ software is a discrete element method to study the characteristics of medium by simulating the motion and interaction of granular media [20]. It uses discontinuous numerical method to solve practical problems with complex deformation conditions. Therefore, it is widely adopted in geotechnical engineering, geological engineering, and mining engineering [21, 22]. Parallel bond (PB) and contact bond $(\mathrm{CB})$ are defined in PFC to illustrate the relationship between model particles. PB can transfer both force and bending moment, and it is suitable to simulate dense materials such as rock and coal [23]. Hence, it was adopted in this study.

3.1. Model Building. In this study, two mining schemes were designed. No DHB is used in Scheme 1, while seven groups of roof blasting boreholes were arranged along the direction of advanced roadway in Scheme 2. The drilling parameters in numerical simulation were consistent with the engineering sites. Based on the two schemes, a 2D numerical model was established along the inclination and strike of the working face (Figure 4). The positions of the inclination model and strike model were selected at the axial section of the blasting borehole and at the charge section of the blasting borehole, respectively.

The inclination model established according to the actual engineering conditions was $210 \mathrm{~m}$ in length, $70 \mathrm{~m}$ in width, and $4.5 \mathrm{~m} \times 5 \mathrm{~m}$ in roadway section size, while the strike model was $160 \mathrm{~m}$ long and $70 \mathrm{~m}$ wide. The caving hydraulic support was imported into PFC by Rhino modeling software, and the maximum support height of the hydraulic support was $4.8 \mathrm{~m}$.

3.2. Meso-Mechanical Parameters. In the PB model, macroscopic mechanical parameters such as compressive strength and elastic modulus are represented by parallel bond modulus, parallel bond stiffness ratio, normal bond strength, and tangential bond strength. Appropriate mesoscopic parameters are crucial to obtaining reasonable numerical results. In the 


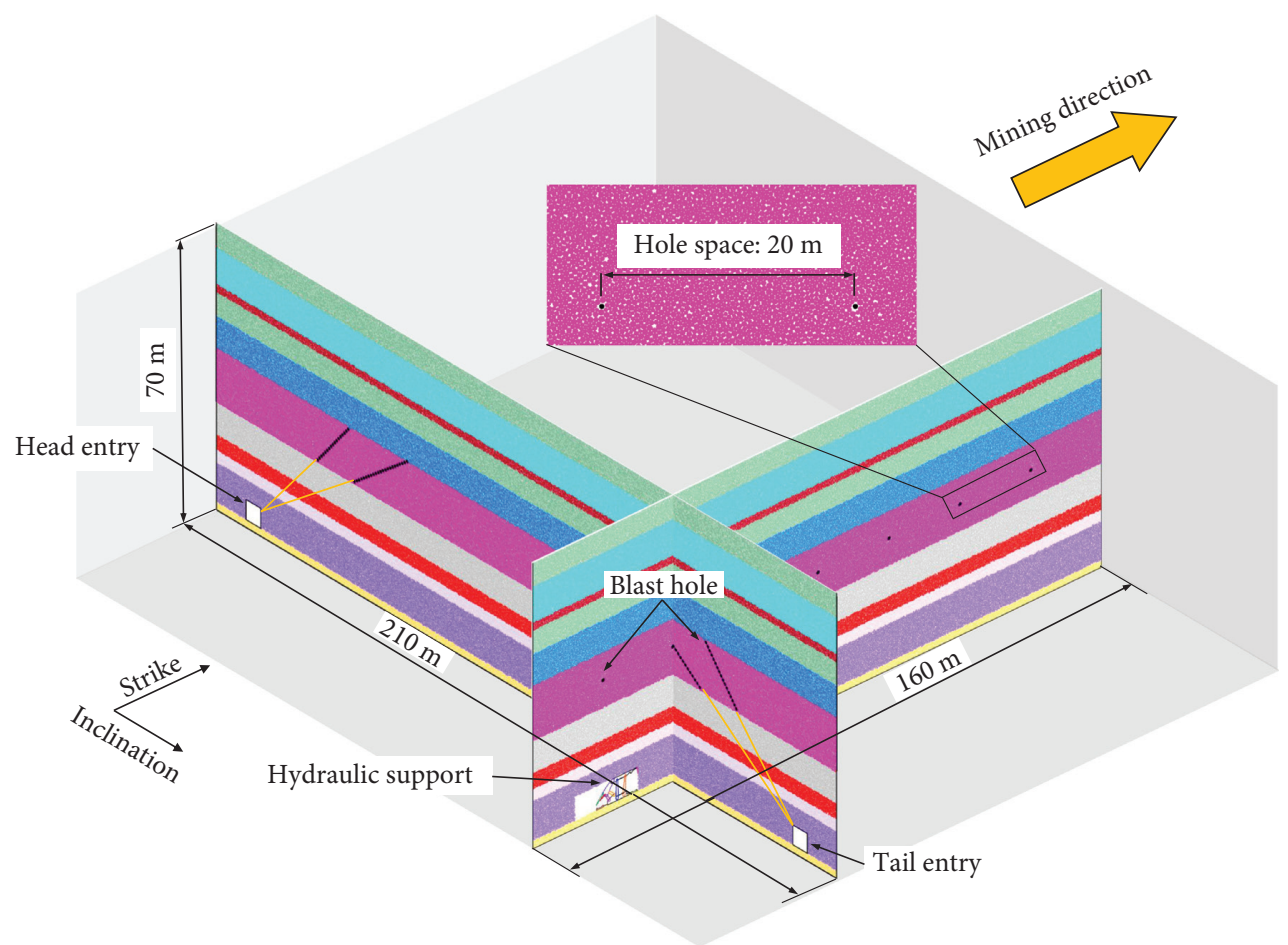

FIgURE 4: Diagram of strike and dip models and blasting drilling.

preliminary work of this study, rock cores of $4 \#$ coal seam and its roof and floor were drilled from the roadway of the 302 working face and processed into standard samples according to ISRM requirements. Uniaxial compression test was conducted by MTS electrohydraulic servo testing machine in the laboratory with constant displacement loading to obtain the macroscopic mechanical parameters and stress-strain curves. Then, the numerical model of coal rock consistent with the real sample was established, and the meso-mechanical parameters were continuously adjusted by the trial and error method until the test results basically conform to those of the real coal rock sample. The stress-strain curves and failure modes are compared in Figure 5. The meso-mechanical parameters of coal rock obtained by parameter calibration are shown in Table 1.

3.3. Blast Loading. The particle expansion method in PFC is used to simulate the failure effect of blasting in rock (Figure 6). First, the explosive coil is simplified into a circular particle with an initial radius of $r_{0}$. Then, the blasting process of explosives is simulated by instantaneous expansion of the particle radius. When the radius of the charge roll expands to the radius of the blast hole, it contacts and interacts with the rock particles around the blast hole. According to the particle contact principle [24], the pressure $P$ acting on the hole wall particles will generate radial thrust on the surrounding rock particles. The expression of the thrust $F$, the particle expansion $d_{r}$ and the normal stiffness $K_{n}$ of the particles is

$$
F=K_{n} d_{r}=2 \pi r_{0} P
$$

Under the action of concentrated cylindrical charge, the explosion stress wave, which usually equals the pulse stress wave [25], propagates outward in the form of spherical wave from the center of charge. In this study, it is simplified as half-sine wave. The expression is

$$
p(t)=\frac{A}{2}[1-\cos (2 \pi f t)],
$$

where $p(t)$ is gas expansion pressure, $\mathrm{MPa} ; A$ is the peak pressure of blasting hole wall, MPa; $f$ is the frequency of half-sine wave, $\mathrm{Hz}$; and $t$ is the duration of half-sine wave, s.

3.4. Dynamic Boundary Conditions. Considering that the research object is near-field semi-infinite space, it is necessary to set the diffusion boundary of stress wave to absorb the kinetic energy of incident wave to simulate the infinite medium [26]. In this study, the contact force is applied to the model boundary particles to realize the transmission boundary, and the diffusion effect of stress wave is also considered [25].

3.5. Stepped Excavation. After the initial model establishment and $\mathrm{DHB}$, the strike and inclination models in Schemes 1 and 2 were excavated, respectively. The inclination model was excavated once, and the excavation range was $190 \mathrm{~m}$ of the inclination length of the working face. 


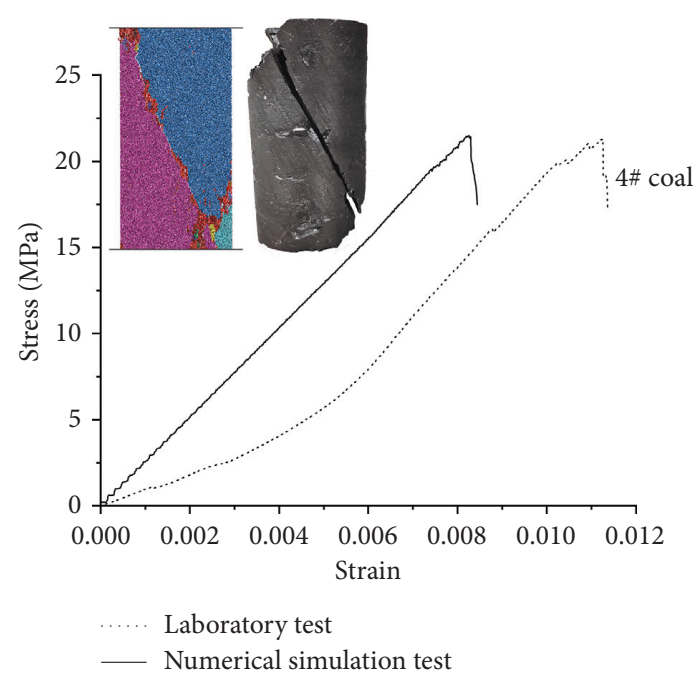

(a)

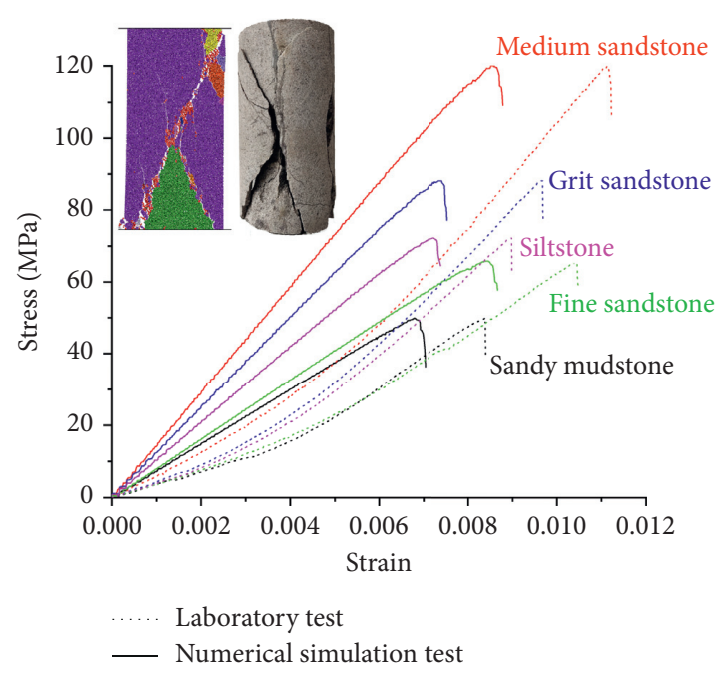

(b)

FIgURE 5: Calibration curves of the parameters of coal rock mass. (a) 4\# coal seam. (b) Surrounding rock.

TABLE 1: Mesoscopic mechanical parameters of the particle model.

\begin{tabular}{|c|c|c|c|c|c|c|c|c|c|c|}
\hline Lithology & $\begin{array}{l}\text { Minimum } \\
\text { particle } \\
\text { radius }(\mathrm{mm})\end{array}$ & $\begin{array}{c}\text { Particle } \\
\text { radius } \\
\text { ratio }\end{array}$ & $\begin{array}{l}\text { Particle } \\
\text { density } \\
\left(\mathrm{kg} / \mathrm{m}^{3}\right)\end{array}$ & $\begin{array}{c}\text { Particle } \\
\text { contact } \\
\text { modulus } \\
(\mathrm{GPa})\end{array}$ & $\begin{array}{l}\text { Normal } \\
\text { tangential } \\
\text { stiffness } \\
\text { ratio } \\
\end{array}$ & $\begin{array}{l}\text { Particle } \\
\text { friction } \\
\text { coefficient }\end{array}$ & $\begin{array}{l}\text { Parallel } \\
\text { bond } \\
\text { modulus } \\
(\mathrm{GPa})\end{array}$ & $\begin{array}{l}\text { Parallel } \\
\text { bond } \\
\text { stiffness } \\
\text { ratio } \\
\end{array}$ & $\begin{array}{l}\text { Normal } \\
\text { bond } \\
\text { strength } \\
(\mathrm{MPa})\end{array}$ & $\begin{array}{l}\text { Shear } \\
\text { bond } \\
\text { strength } \\
(\mathrm{MPa})\end{array}$ \\
\hline Siltstone & 100 & 1.66 & 2600 & 4.2 & 1.6 & 0.25 & 4.2 & 1.6 & 8.8 & 17.3 \\
\hline $\begin{array}{l}\text { Sandy } \\
\text { mudstone }\end{array}$ & 100 & 1.66 & 2500 & 1.8 & 1.6 & 0.25 & 1.8 & 1.6 & 5.5 & 15.6 \\
\hline $\begin{array}{l}\text { Grit } \\
\text { sandstone }\end{array}$ & 100 & 1.66 & 2550 & 5.0 & 1.8 & 0.25 & 5.0 & 1.8 & 10.1 & 18.5 \\
\hline $\begin{array}{l}\text { Medium } \\
\text { sandstone }\end{array}$ & 100 & 1.66 & 2500 & 9.1 & 1.7 & 0.25 & 9.1 & 1.7 & 15.2 & 27.5 \\
\hline $\begin{array}{l}\text { Fine } \\
\text { sandstone }\end{array}$ & 100 & 1.66 & 2500 & 2.6 & 1.5 & 0.25 & 2.6 & 1.5 & 7.5 & 18.8 \\
\hline Mudstone & 70 & 1.66 & 2500 & 2.5 & 1.4 & 0.25 & 2.5 & 1.4 & 5.6 & 12.0 \\
\hline $\begin{array}{l}4 \# \text { coal } \\
\text { seam }\end{array}$ & 50 & 1.66 & 1350 & 0.8 & 1.2 & 0.25 & 0.8 & 1.1 & 3.5 & 6.3 \\
\hline $\begin{array}{l}\text { Carbon } \\
\text { mudstone }\end{array}$ & 70 & 1.66 & 2500 & 1.4 & 1.4 & 0.25 & 1.4 & 1.4 & 4.4 & 9.1 \\
\hline
\end{tabular}

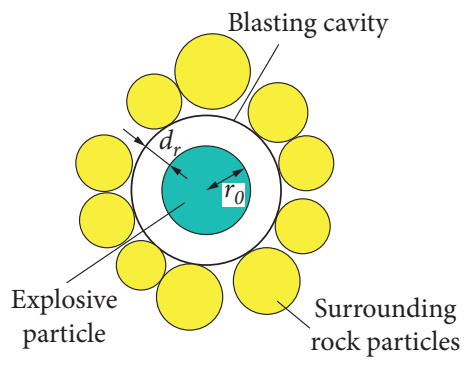

(a)

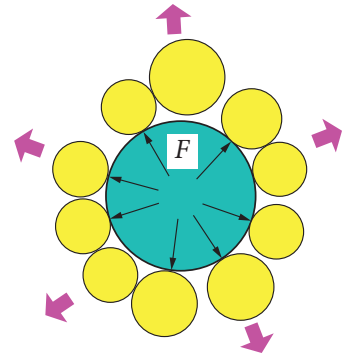

(b)

Figure 6: Schematic diagram of particle expansion. (a) Initial state. (b) Expansion state. 
Stepped excavation was adopted in the strike model, with $10 \mathrm{~m}$ for each excavation and $140 \mathrm{~m}$ for cumulative excavation. During mining, the hydraulic support gradually moves forward following the working face.

\section{Result Analysis}

4.1. Distribution of Damage Range. The process of rock blasting and breakage is mainly controlled by two actions, namely, dynamic action of shock wave and quasi-static action of detonation gas [27]. After the application of roof DHB in Scheme 2, the distributions of single-hole damage range extracted along the radial and axial directions of the borehole are shown in Figures 7(a) and 7(b). The blastinginduced single-hole damage range is generally characterized by annular zoning. (1) Crushed zone: under the tensile effect of shock wave, nearly all the contact keys between rock particles near the blasting borehole are destroyed, indicating that the stress acting on the rock particles around the borehole wall exceeds the tensile or shear strength of the contact keys. The damage in this zone is mainly tangential tensile fracture, and the diameter of crushed zone is in the range of $0.62 \mathrm{~m}-0.71 \mathrm{~m}$. (2) Crack zone: the crack zone lies beyond the crushed zone. The damage in this zone is dominated by several radially extended banded tensile cracks, and the diameter of this zone is in the range of 2.01-2.23 m. (3) Elastic vibration zone: the elastic vibration zone, which is affected by residual detonation gas, lies beyond the crack zone. Rock particles just vibrate without obvious cracks. Therefore, the effective failure diameter of single-hole blasting based on numerical simulation is in the range of $2.01-2.23 \mathrm{~m}$.

4.2. Evolution of Spatial Structure of Overlying Strata. The theory of mine pressure and strata movement has always been the core scientific focus of mining. The dynamic evolution of overburden spatial structure along with mining will be the main control factor of hard-roof rock burst $[28,29]$. Therefore, the dynamic evolution characteristics of overburden spatial structure with mining in the two simulation schemes were analyzed.

According to Figure 8 (a-60 m), the working face is in the first weighting stage. When roof DHB is not adopted, after the coal seam is mined, the immediate roof enters the collapse zone. However, the medium sandstone key stratum does not break for its great thickness, high strength, and good stability. Only a small amount of longitudinal tensile breakage occurs in the lower part of the key strata. Overall, it can still be regarded as a solid support. An obvious "triangle" separation layer occurs between the key strata and the underlying strata, providing space for the subsequent impact subsidence of hard rock strata and presenting a long-distance " $F$ " overburden structure. In Figure 8 (a-80 $\mathrm{m}$ and $\mathrm{a}-100 \mathrm{~m}$ ), with the further increase in the gob area, the medium sandstone key stratum undergoes shear sliding instability, and massive accumulated elastic energy is released instantaneously. The key stratum breaks into longdistance rock blocks $\mathrm{A}$ and $\mathrm{B}$, which form an unstable weak hinge structure. With the advancement of the working face, the hinged structure fails and the secondary sliding instability occurs, which leads to the synchronous breakage and collapse of the overlying strata within a certain range. Meanwhile, the crack zone further develops along the longitudinal direction. The hanging roof of the key strata at the edge of the gob will again form a long-distance " $F$ " overburden structure and begins to accumulate energy for the next round of sliding instability of the key strata. In Figure 8 (a-120 $\mathrm{m}$ and a-140 $\mathrm{m}$ ), after rock block $\mathrm{C}$ in the high-level key strata reaches the ultimate collapse distance, sliding instability occurs and the overlying strata are separated again, which provides space for sliding instability of the higher-level strata. The collapse of subsequent overlying strata will repeat in the form of periodic weighting.

During mining, due to the insufficient collapse of the key strata, the static load stress of coal rock around the stope jumps. Due to the large area of the first breakage, each breakage of the key strata and the overlying strata will cause strong vibration, forming high impact dynamic load on the support and roadway below. The low-level separation layer that has not been fully compacted under the key strata is the area suffering frequent occurrence of middle- and smallenergy seismicities. Since the seismic source is closer to the working face roadway and the rock activities are more frequent, the seismicities will exert multiple rounds of cyclic impact disturbance on the roadway. Therefore, the elastic energy accumulated in the hanging roof is superimposed with the strong dynamic load released by the breakage of high-level Key strata and the middle- and small-energy dynamic loads released by the breakage of low-level strata. Once it exceeds the ultimate bearing strength of roadway surrounding rock, large-scale mine seismicities or hard-roof rock burst will be induced.

According to Figure 8 (b-60 m), the working face is also in the first weighting stage. After the application of roof DHB, cracks with different directions and consistent characteristics are generated around the blasting boreholes in each group. Although the cracks are of limited lengths, the key strata do not break. Due to the existence of blasting-induced cracks, few longitudinal tensile cracks develop to the top of the model in the middle of the gob, that is, the position of the largest bending moment of the fixed support beam. The key stratum experiences a certain bending subsidence, which first deteriorates the energy storage and bearing capacity of the main roof. According to Figure 8 (b-80 m and b-100 m), due to the existence of blasting-induced cracks, as the working face further advances, the longitudinal cracks around the blasting borehole become channels for gradual breakage of the medium sandstone key strata and its overlying strata. Fractures with different scales and directions between adjacent groups of blasting boreholes develop and connect with each other. The key stratum does not experience instantaneous shear sliding instability, but it subsides slowly by the synergetic breakage of blocks A, B, and C. The long-distance "F" overburden structure changes into a more stable short-distance "F" overburden structure. The key stratum collapses more fully; the separation space is further compressed, and the relative movement between the broken blocks tends to 


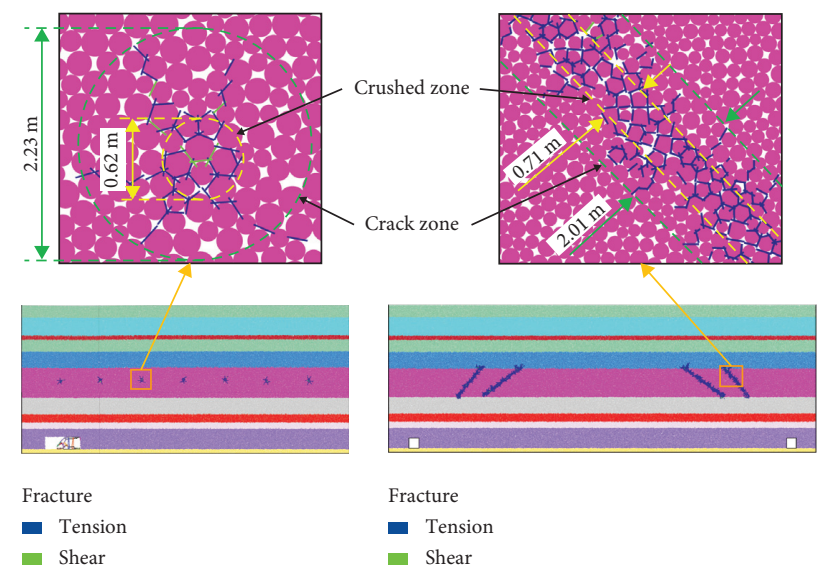

FIgURE 7: Ranges of damage zones induced by deep-hole blasting. (a) Strike, (b) Inclination.

stabilize. According to Figures 8 (b-120 m and b-140 m), with the continuous advance of the working face, the medium sandstone key strata and its overlying strata will gradually break stably along the longitudinal cracks induced by DHB. The breakage morphology of the key strata with mining will change from long-distance, integrated strata and instantaneous sliding instability to short-distance, stratified strata and slow synergetic subsidence.

Figures 9(a) and 9(b) illustrate the spatial structure of overlying strata after working face recovery in the two schemes. Under the action of concentrated cylindrical charge blasting, the fracture distance of the key strata along the inclination of the working face also plunges, from longdistance rock blocks A, B, C, D, E, and F to relatively shortdistance rock blocks A, B, C, D, E, F, G, and $\mathrm{H}$. The cutting effect of the key strata along the axial direction of the blasting borehole is especially obvious in the area of borehole charge section. Meanwhile, due to the full collapse of the overlying roof, the separation layer between the key strata and its underlying strata is obviously compressed, and the reduction of the separation space also avoids the impact subsidence of the high-level key strata, thus suppressing the occurrence of hard-roof rock burst.

4.3. Distribution of Force Chain. In a discontinuous discrete medium, friction and contact occur between rock particles along the normal direction and the tangential direction under the action of external load, causing changes in the internal load of the granular material [30, 31]. The thickness and density of force chains, which reflect the macroscopic force state of an object, can be used to characterize the transformation of the bearing mode of overlying strata in the process of mining [32].

4.3.1. Characteristics of Force Chain Network. The distribution and development of the force chain structure network in the strike and inclination models with the advancement of the working face are given in Figures 10 and 11.

The force chain structure network of the initial rock stratum is evenly distributed. Under the action of external load, almost all the properties of the force chain structure are in a compressed state. The difference in mechanical properties leads to natural stratification of the force chain structure network between different strata. Mining activities disrupt the balance of the initial force chain structure. As a result, the force chain structure network is distributed in a complex mode.

As illustrated in Figure $10(a-40 \mathrm{~m})$, the working face is in the stage of first weighting. In the absence of roof DHB, the immediate roof breaks directly into the gob after coal seam mining. Resultantly, the contact keys between rock particles are damaged, and most of force chains fail, which leads to a significant decrease in the strength and density of force chains of the collapsed rock mass. However, the key strata of the gob have not been broken. Affected by the bending moment of the fixed beam, the force chain structure of the particles in the middle and lower parts of the key rock beam is in a tensile state. In contrast, affected by the extrusion of the overlying high-level rock stratum and the clamping action of horizontal load, the middle and upper parts of the key rock beam are in a compressive state. Overall, the key rock beam presents an arched structure that is compressed in the upper part and tensile in the lower part (hereafter referred to as CUTL). In the longitudinal direction, all the rock strata bear the load from upper strata in similar arched structures. In the horizontal direction, the hanging medium sandstone key stratum transmits the load to the fixed support end, and strong chains are highly concentrated and act on the hydraulic support. In this case, the main risk threatening the working face is the high pressure on the hydraulic support. In Figure 10 (a-60 m and a- $80 \mathrm{~m}$ ), due to the disturbance of mining, the overall force chain density of the surrounding rock rises, and the strong force chains in front of the coal wall of the working face become increasingly concentrated in a larger range. Meanwhile, the strata that have not collapsed in the gob also experience obviously concentrated distribution of compressive force chains, and the density and strength of force chains reach their peaks as a result of the squeezing of rock mass particles. Local breakage of the key strata starts from the tensile force chain zone in the middle and lower parts. As the range of the gob expands, the key stratum gets completely broken and drives the overlying strata to break 
(a-60 m)

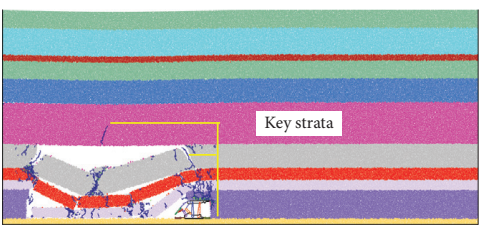

Fracture

Tension

Shear

(a-80 m)

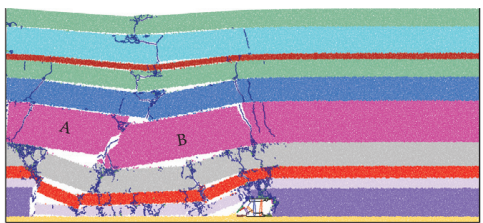

Fracture

Tension

Shear

(a-100 m)

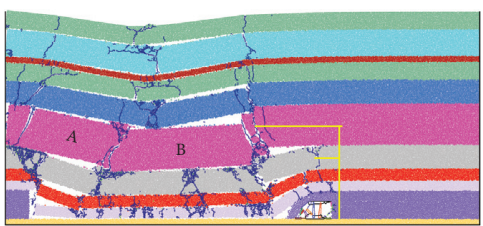

Fracture

Tension

Shear

(a-120 m)

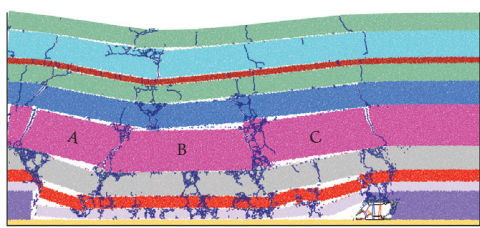

Fracture

- Tension

Shear

(a-140 m)

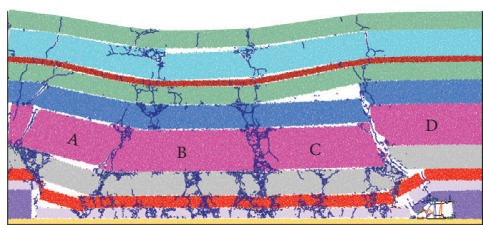

Fracture

Tension

Shear

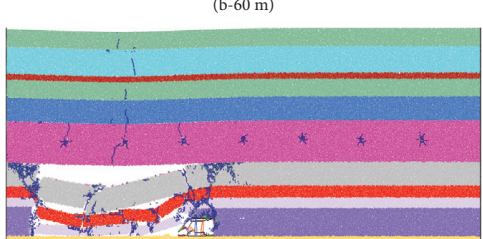

Fracture

Tension

Shear

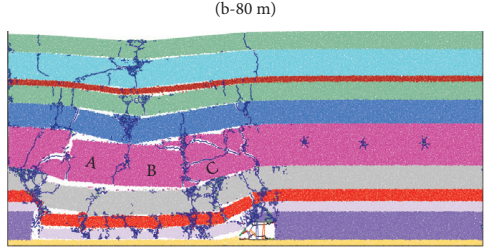

Fracture

Tension

Shear

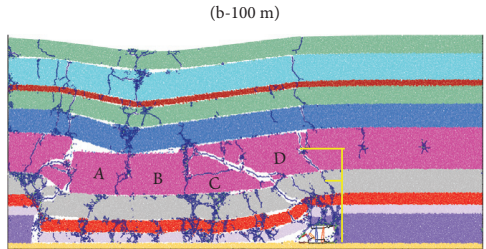

Fracture

Tension

Shear

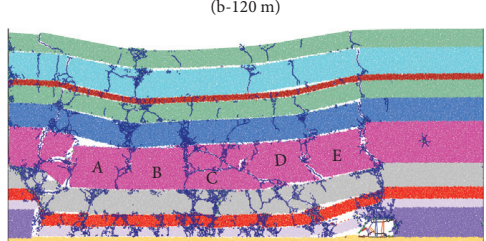

Fracture

Tension

Shear

(b-140 m)

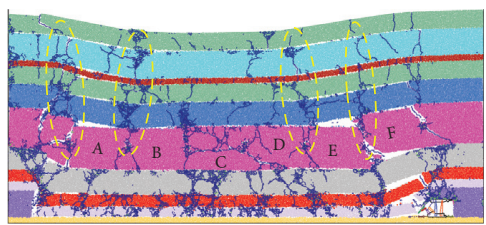

Fracture

Tension

Shear

FiguRE 8: Overburden rock breakage and fracture development during working face recovery.

synchronously. The original rock beam arched structure is destroyed. The rock blocks that break into sections overlap naturally after subsiding, stress becoming concentrated at the position of gangue contact. At this moment, unstable weak force chains dominate. In Figure 10 (a-100 m and a-140 m), when the working face continues to advance, the range of CUTL arched strong chains expands further, and the hanging roof above the working face turns into a force chain structure that is tensile in the upper part and compressed in the lower part (hereafter referred to as TUCL). The key stratum accumulates elastic energy again and transmits it to the front of the working face. The evolution characteristics of the subsequent force chain structure will repeat in the form of periodic weighting. Therefore, the 


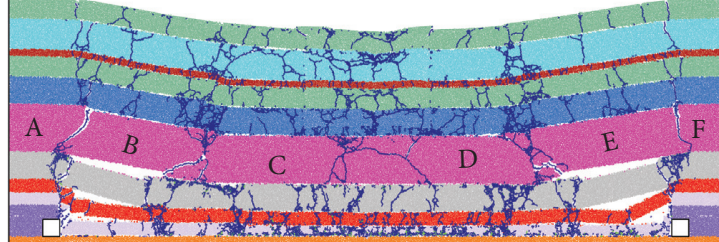

Fracture

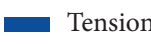

Shear

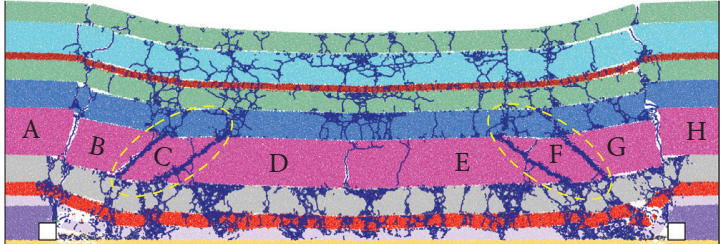

Fracture

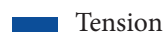

Shear

(a)

(b)

FIGURE 9: Overburden collapse and fracture development along the inclination of working face. (a) Nonblasting. (b) Blasting.

existence of hard thick rock strata will result in the alternate appearance of two force chain structures, namely, "CUTL" in the middle of the gob and "TUCL" at the edge of the gob, and the static load stress concentration near the roadway always remains relatively high.

As shown in Figure 10 (b-40 m and b-60 m), after the application of $\mathrm{DHB}$, rock particles near the blasting hole are almost completely destroyed, which results in the formation of a force chain cavity in the crushed zone. Due to the squeezing and expansion of gases generated by the blasting, the force chain structure in the crack zone is concentrated in a circle. With the advancement of the working face, the range of the original cavity above the gob continues to expand. Although the force chain structure of the key strata is still roughly CUTL, the destructive effect of the blasting hole weakens the concentration of the force chain structure. Consequently, the influence range of advance bearing pressure of the working face is significantly reduced. In Figure 10 (b-80 m and b-100 m), with the further advancement of the working face, the original internal cracks induced by DHB gradually develop and expand, driving the generation of a connected fracture belt along the longitudinal direction in the key strata and their overlying strata. As a result, in terms of the sizes of blocks, the strata become increasingly fragmented; on the other hand, this also effectively weakens the force chain structure that is "CUTL" in the middle of the gob and "TUCL" at the edge of the gob. In Figure 10 (b-120 $\mathrm{m}$ and b-140 m), as the working face advances further, the arched force chain structure still moves forward with its basic form, but the range does not significantly expand. The density and strength of force chains both plunge, and the static load stress concentration near the roadway also remains relatively stable.

The distributions of the force chain structure on the inclination after the recovery of the working face under the two schemes are displayed in Figures 11(a) and 11(b), respectively. When deep-hole blasting is not adopted for roof treatment, insufficient collapse in the gob causes the blocks to be staggered to form a strong force chain network. In contrast, affected by the extrusion of the overlying stratum and the clamping action of horizontal load, the key strata blocks in the middle of the working face present a force chain structure that is "TUCL." The key strata that has not fully collapsed at the end of the working face transmits the additional load of the overlying strata to the top of head entry and tail entry, resulting in severe stress concentration in the roadway roof and the coal body. After DHB is adopted, the low-level rock strata in the gob will collapse more completely, dominated by a uniformly distributed weak-force chain structure. As the key stratum breaks into more small blocks, the original force chain structure, that is, TUCL, changes into a relatively stable and uniform distribution. The cantilever beam at the edge of the gob is shortened, and the stress concentration around the roadway is effectively weakened.

4.3.2. Characteristics of Compression Force Chain Strength. The force chain structure network undertakes the contact force between rock particles [33]. The medium sandstone key stratum, in which two force chain types (i.e., compression and tension) exist, is anisotropic with a complex spatial distribution. The number of force chains changes with the breakage and movement of the key strata. The compression and tension of blocks cause varying degrees of changes in the strength of the force chains. To quantitatively characterize the strength evolution characteristics of compressive force chain of strata in the mining process, this study proposes for the first time to define the compressive force chain strength (CFCS) to be the ratio of the sum of contact forces of compressive force chains between rock particles to the total number of compressive force chains. The CFCS can be calculated by

$$
P=\frac{1}{S} \sum_{i=1}^{S} f_{i},
$$

where $P$ is defined as the CFCS; $f_{i}$ is the magnitude of the contact force in the $i$ th compressed force chain; and $S$ is the total number of compressive force chains in a specific rock stratum. According to equation (3), the CFCSs of the rock particles in the key strata in different stages of working face recovery in the two schemes can be calculated and plotted (Figure 12).

As can be observed from the red curve in Figure 12, in the nonblasting scheme, at the beginning of working face recovery (Stage I), the CFCS of the roof key strata particles keeps going up, reaching the peak value (about $280 \mathrm{kN}$ ) at the recovery step of $73 \mathrm{~m}$. Afterwards, the CFCS keeps rising 
(a-40 m)

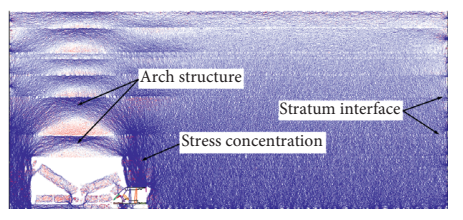

Contact force chain

Compression

Tension

(a-60 m)

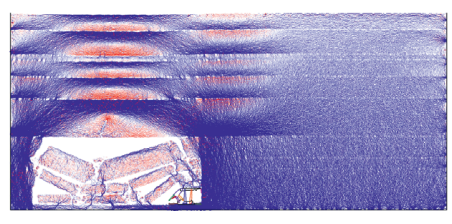

Contact force chain

Compression

Tension

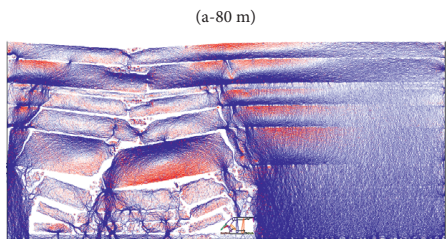

Contact force chain

Compression

Tension

(a-100 m)

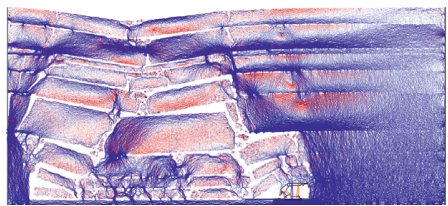

Contact force chain

Compression

Tension

(a-120 m)

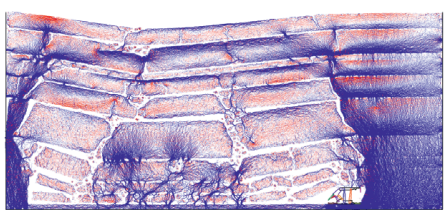

Contact force chain

Compression

Tension

(a-140 m)

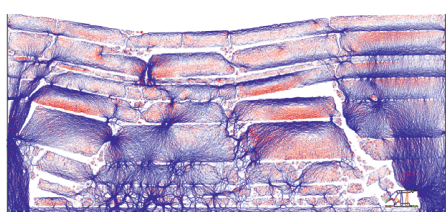

Contact force chain

Compression

Tension (b-40 m)

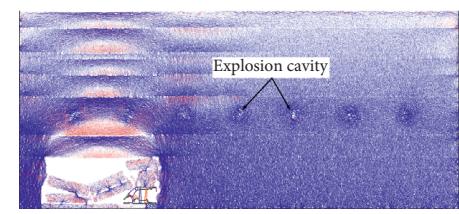

Contact force chain

Compression

Tension

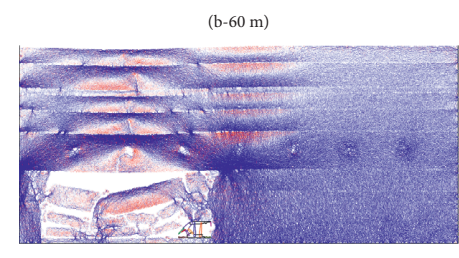

Contact force chain

Compression

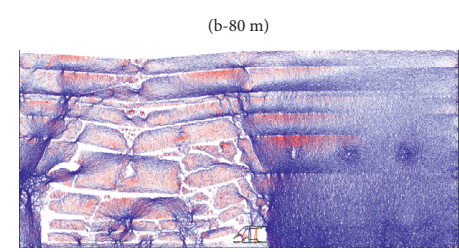

Contact force chain

Compression

Tension

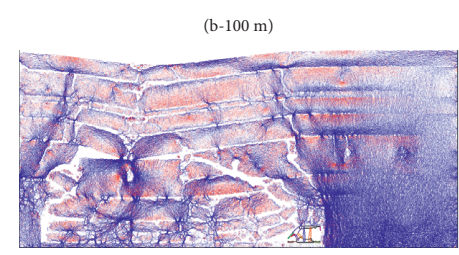

Contact force chain

Compression

Tension

(b-120 m)

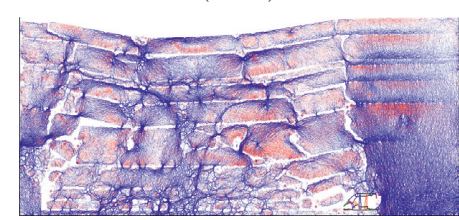

Contact force chain

Compression

Tension

(b-140 m)

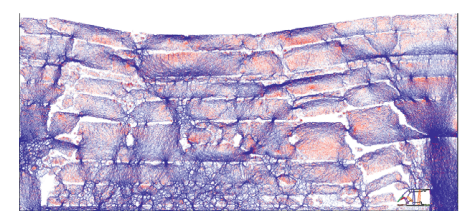

Contact force chain

Compression

Tension

Figure 10: Development of force chains during the advancement of the working face. 


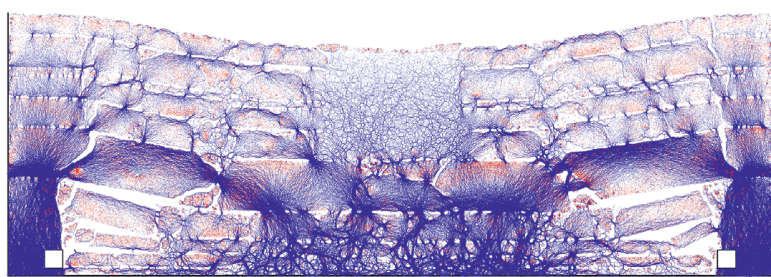

Contact force chain
Compression
Tension

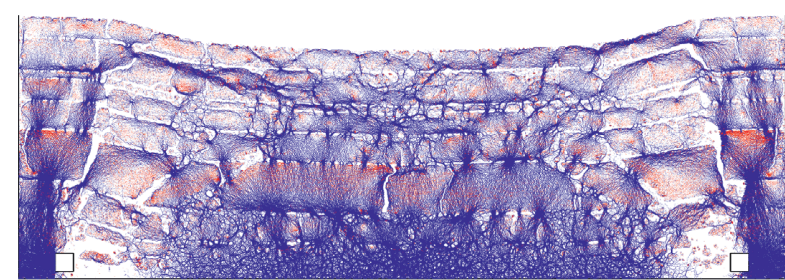

Contact force chain

Compression Tension

(a)

(b)

FIGURE 11: Development of the force chain on the inclination of the working face. (a) Nonblasting. (b) Blasting.

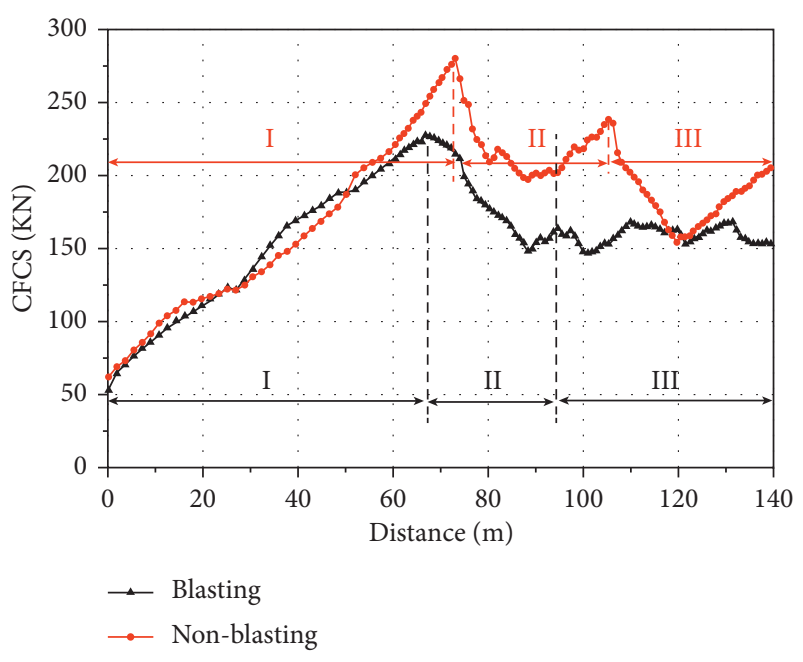

FIgURE 12: Variation curves of CFCS in the key stratum.

and falling cyclically (Stages II and III), the maximum change being about $85 \mathrm{kN}$. Such cyclical changes roughly correspond to the generation and breakage of hanging roof in the key strata. Therefore, in the absence of DHB, the CFCS is of a great peak value and a large subsequent periodic change amplitude.

As can be seen from the black curve in Figure 12, in the blasting scheme, in Stage I, the CFCS increases in a basically consistent trend with the red curve. The differences are that the peak value is only $230 \mathrm{kN}$, which is $17.85 \%$ lower than that in the nonblasting scheme, and the recovery step corresponding to the peak value is also reduced by about $8 \mathrm{~m}$. After that, the CFCS plunges (Stage II) by about $85 \mathrm{kN}$ as well. Later, in Stage III, the CFCS gradually stabilizes, fluctuating in the range of $150-165 \mathrm{kN}$. The above results suggest that roof DHB remarkably decreases the peak value and periodic change amplitude of CFCS in the key strata and weakens the ability of the key strata to bear and transmit load. It can be concluded that DHB can effectively control the strong static load and dynamic load of hard-rock rock burst.

4.4. Pressure Monitoring Results of Hydraulic Support. The step distance of roof weighting and the pressure anomaly during periodic weighting can be determined by analyzing the real-time pressure monitoring results obtained by the hydraulic support in the mining process. In this study, the compressive force of overburden particles in contact with the top beam of hydraulic support is used to characterize the working resistance of hydraulic support in the mining process. The relative position relationship between the top beam of hydraulic support and the overburden particles is illustrated in Figure 13, and the monitoring curves of the working resistance of hydraulic support in different mining stages of working face recovery in the two schemes are shown in Figure 14.

As exhibited by the red curve in Figure 14, in the nonblasting scheme, the curve is obviously oscillated as a result of multiple slipping and breakage of the inadequately collapsed key strata and their underlying strata. After working face recovery starts, the working resistance of the hydraulic support keeps rising, reaching the peak value (about $21.5 \mathrm{MN}$ ) at the step distance of the first weighting (about $72 \mathrm{~m}$ ). Then, the working face enters the periodic weighting stages (Stages I and II). In each periodic weighting stage, the working resistance curve maintains high stress for a while first because the length of hanging roof has not reached the ultimate collapse distance, and then it drops due to the breakage of hanging roof. Afterwards, it rises again in the next stage. The step distances of periodic weighting in Stages I and II are $31.5 \mathrm{~m}$ and $36.6 \mathrm{~m}$, respectively. Such a variation trend occurs repeatedly in the subsequent curve, which features "a large step distance, many oscillations, and a long duration."

As displayed by the black curve in Figure 14, in the blasting scheme, the working resistance curve shares a basically consistent growth trend with the red curve in the initial stage of working face recovery, but the oscillations are notably weakened. The peak value of the working resistance and step distance of the first weighting are $17 \mathrm{MN}$ and about $67.5 \mathrm{~m}$, which are about $20.9 \%$ and $6.25 \%$ smaller than those in the nonblasting scheme, respectively. Afterwards, within the recovery step, two periodic weighting stages appear in the nonblasting scheme, while the number becomes three in the blasting scheme. In each periodic weighting stage, because DHB avoids the generation of hanging roof in the key strata, the original variation trend (i.e., the load remains high due to the generation of hanging roof, falls due to the breakage of hanging roof, and keeps rising again later) weakens and turns into another feature (i.e., a large step 


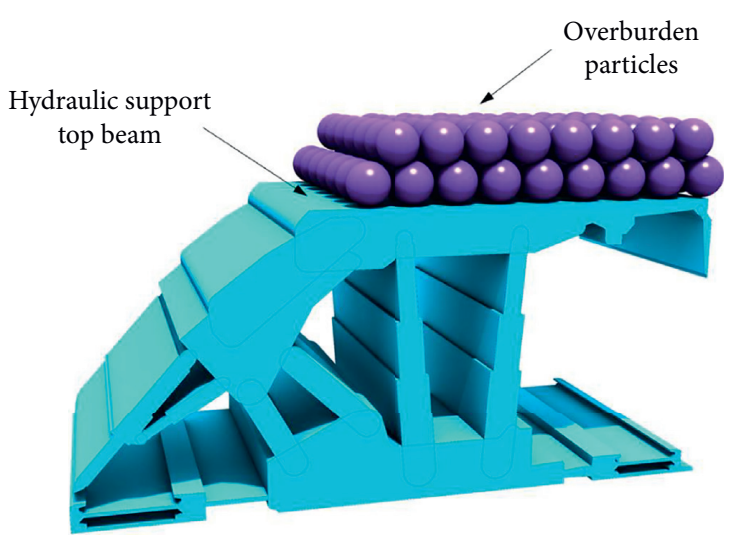

Figure 13: Hydraulic support and the overburden particles.

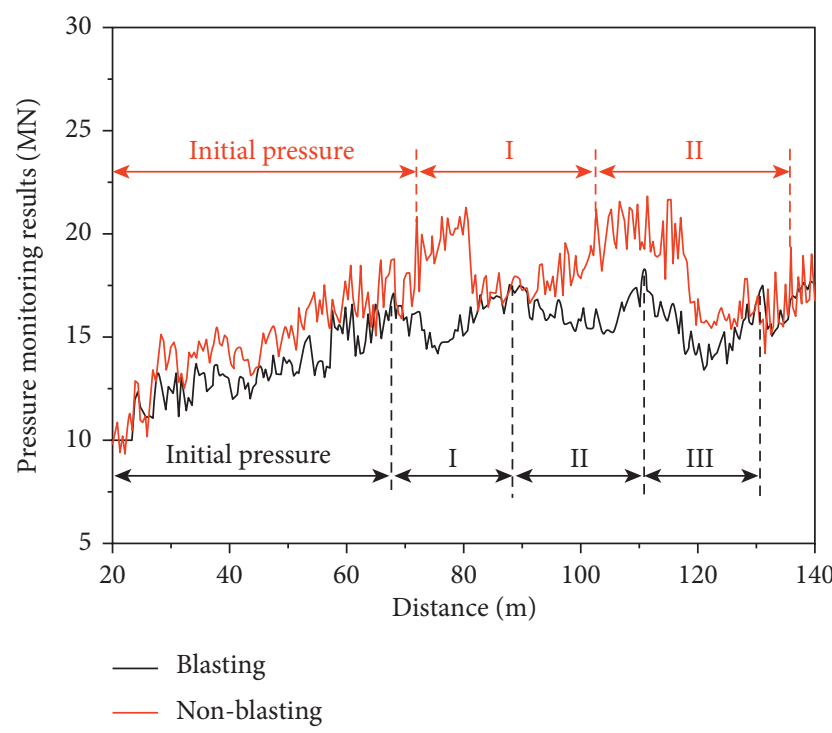

FIgURE 14: Monitoring curves of the working resistance of hydraulic support.

distance, many oscillations, and a long duration). The step distances of periodic weighting in Stages I, II, and III are $21.5 \mathrm{~m}, 23.2 \mathrm{~m}$, and $22.7 \mathrm{~m}$, respectively, which are shortened by about $32 \%$ on average. The step distances of periodic weighting are quite close to spacing between different groups of boreholes $(20 \mathrm{~m})$. This demonstrates that the roof DHB technology effectively shortens the breakage step distance of the key strata and boasts a significant effect on controlling hard-roof rock burst.

\section{Discussion}

5.1. Effect of Static Stress Reduction. The original rock in the deep stope is of relatively high stress. The existence of hard thick roof enables the additional load of the overlying strata to be transmitted to the coal rock around the roadway. Resultantly, the bearing pressure corresponds to a greater peak value which occurs closer to the roadway surface. The two force chain structures, namely, "CUTL" in the middle of the gob and "TUCL" at the edge of the gob, appear alternately, and the static load stress concentration near the roadway always remains relatively high. After DHB is adopted, the hard thick roof is no longer integrated and stable. Accordingly, the breakage distances on the strike and inclination of the working face are both shortened, and the density and strength of force chains are effectively reduced. Besides, the continuity and transmission path of the arched force chain structure of overburden are also obviously damaged. The monitoring curve of the working resistance of hydraulic support shows that roof DHB can effectively alleviate the bearing situation above the stope of the working face and keep the static load stress level and the fluctuation range at a low level.

5.2. Effect of Dynamic Stress Reduction. Since the hard thick roof is of a longer breakage distance and greater accumulated elastic energy, when it loses stability, the seismic source will fracture in a wider range at a higher speed, releasing higher dynamic load. In addition to the transient stress increase, the dynamic load caused by the slippage breakage of the hard thick roof also includes the cyclic loading and unloading of coal rock. When the dynamic stress wave caused by the breakage of the key strata acts on the coal rock that is already in the elastic-plastic composite zone, the roof will subside once instability occurs. Consequently, the roadway will be instantly blocked by the coal body washed out from the side. The monitoring, early warning, and prevention and control of hard-roof rock burst are rather difficult because the magnitude of dynamic stress and the location of the seismic source are difficult to determine. The application of roof DHB can significantly improve the collapse morphology of roof overburden, reduce the separation space between strata, and thus avoid the impact subsidence of high-level strata. The monitoring curves of the working resistance of hydraulic support indicate that roof DHB can effectively shorten the step distance of periodic weighting and reduce the variation amplitude of the overlying bearing load of the stope in the mining process, thereby ensuring that the dynamic load of roof breakage is in a relatively controllable range.

\section{Conclusions}

In this study, the disaster-causing mechanism of hard-roof rock burst was investigated based on the mechanism of rock burst induced by the coupling of dynamic load and static load. Specifically, the mechanism of hard-roof rock burst control by DHB was researched on in depth from four aspects, namely, the damage range of blasting boreholes, the temporal and spatial evolutions of overlying strata, and the working resistance of hydraulic support. The main conclusions are as follows:

(1) The technical scheme of roof DHB along the strike and inclination of the working face was simulated with the aid of PFC2D software, and the point and concentrated cylindrical charge blasting processes were simulated by using the particle expansion method, which provides a new means for the numerical analysis of roof DHB technology. 
(2) The numerical simulation results show that roof DHB changes the breakage morphology of the key strata. The continuity and transmission path of the overburden arched force chain structure are destroyed; the density and strength of force chains are notably reduced; and the load condition around the stope roadway is effectively improved. Moreover, the step distance of periodic weighting is shortened, and the variation amplitude of the overlying bearing load of the stope in the mining process is decreased as well.

(3) The mechanism of hard-roof rock burst control by DHB is reflected by both static load reduction and dynamic load reaction. The research finding provides theoretical support for the subsequent in-depth effect test and parameter optimization of roof deephole blasting.

\section{Data Availability}

The data used to support the findings of this study are available from the corresponding author upon request.

\section{Conflicts of Interest}

The authors declare that they have no conflicts of interest regarding the publication of this paper.

\section{Acknowledgments}

The authors gratefully acknowledge the financial support for this work provided by the National Natural Science Foundation of China (nos. 51874292 and 51934007) and Postgraduate Research and Practice Innovation Program of Jiangsu Province (nos. KYCX21_2349 and KYCX21_2342).

\section{References}

[1] C. G. Zhang, I. Canbulat, B. Hebblewhite, and C. Ward, "Assessing coal burst phenomena in mining and insights into directions for future research," International Journal of Coal Geology, vol. 179, pp. 28-44, 2017.

[2] M. C. He, F. Q. Ren, and D. Q. Liu, "Rockburst mechanism research and its control," International Journal of Mining Science and Technology, vol. 28, no. 5, pp. 829-837, 2018.

[3] Q. X. Qi, Z. H. Ouyang, S. K. Zhao, H. Y. Li, X. L. Li, and N. B. Zhang, "Study on types of rock burst mine and prevention methods in China," Coal Science and Technology, vol. 42, pp. 1-5, 2014.

[4] W. Cai, L. M. Dou, G. Y. Si, and Y. W. Hu, "Fault-induced coal burst mechanism under mining-induced static and dynamic stresses," Engineering, vol. 7, pp. 687-700, 2021.

[5] J. Z. Bai, L. M. Dou, P. Małkowski, J. Z. Li, K. Y. Zhou, and Y. J. Chai, "Mechanical properties and damage behavior of rock-coal-rock combined samples under coupled static and dynamic loads," Geofluids, vol. 2021, Article ID 3181697, 18 pages, 2021.

[6] X. S. Liu, S. L. Song, Y. L. Tan et al., "Similar simulation study on the deformation and failure of surrounding rock of a large section chamber group under dynamic loading,"
International Journal of Mining Science and Technology, vol. 31, no. 3, pp. 495-505, 2021.

[7] J. He, L. M. Dou, A. Y. Cao, S. Y. Gong, and J. W. Lu, "Rock burst induced by roof breakage and its prevention," Journal of Central South University, vol. 19, no. 4, pp. 1086-1091, 2012.

[8] L. M. Dou, J. He, A. Y. Cao, S. Y. Gong, and W. Cai, "Rock burst prevention methods based on theory of dynamic and static combined load induced in coal mine," Journal of China University of Mining and Technology, vol. 40, pp. 1469-1476, 2015.

[9] Z. G. Liu, A. Y. Cao, G. G. Zhu, and C. B. Wang, "Numerical simulation and engineering practice for optimal parameters of deep-hole blasting in sidewalls of roadway," Arabian Journal for Science and Engineering, vol. 42, no. 9, pp. 3809-3818, 2017.

[10] J. W. Liu, C. Y. Liu, and Q. L. Yao, "Mechanisms of crack initiation and propagation in dense linear multihole directional hydraulic fracturing," Shock and Vibration, vol. 2019, Article ID 7953813, 15 pages, 2019.

[11] J. G. Guo, Y. H. Li, F. L. He, G. S. Fu, and S. Gao, "Study on stability control of retained gob-side entry by blasting fracturing roof technology in thick immediate roof," Shock and Vibration, vol. 2021, Article ID 6613562, 2021.

[12] Y. B. Gao, Y. J. Wang, J. Yang, X. Y. Zhang, and M. C. He, "Meso- and macroeffects of roof split blasting on the stability of gateroad surroundings in an innovative nonpillar mining method," Tunnelling and Underground Space Technology, vol. 90, pp. 99-118, 2019.

[13] F. T. Wang, S. H. Tu, Y. Yuan, Y. F. Feng, F. Chen, and H. S. Tu, "Deep-hole pre-split blasting mechanism and its application for controlled roof caving in shallow depth seams," International Journal of Rock Mechanics and Mining Sciences, vol. 64, pp. 112-121, 2013.

[14] P. Konicek, K. Soucek, L. Stas, and R. Singh, "Long-hole destress blasting for rockburst control during deep underground coal mining," International Journal of Rock Mechanics and Mining Sciences, vol. 61, pp. 141-153, 2013.

[15] B. B. Chen, C. Y. Liu, and J. X. Yang, "Design and application of blasting parameters for presplitting hard roof with the aid of empty-hole effect," Shock and Vibration, vol. 2018, Article ID 8749415, 16 pages, 2018.

[16] L. Y. Yang, S. Y. Chen, A. Y. Yang, C. Huang, and H. Z. Xie, "Numerical and experimental study of the presplit blasting failure characteristics under compressive stress," Soil Dynamics and Earthquake Engineering, vol. 149, Article ID 106873, 2021.

[17] M. M. Dehghan Banadaki and B. Mohanty, "Numerical simulation of stress wave induced fractures in rock," International Journal of Impact Engineering, vol. 40-41, pp. 16-25, 2012.

[18] N. K. Meng, Y. Chen, J. B. Bai, X. Y. Wang, W. D. Wu, and B. W. Wu, "Numerical simulation of directional fracturing by shaped charge blasting," Energy Science and Engineering, vol. 8, no. 5, pp. 1824-1839, 2020.

[19] K. Gao, Z. G. Liu, J. Liu et al., "Application of deep borehole blasting to gob-side entry retaining forced roof caving in hard and compound roof deep well," Chinese Journal of Rock Mechanics and Engineering, vol. 32, pp. 1588-1594, 2013.

[20] A. Turichshev and J. Hadjigeorgiou, "Experimental and numerical investigations into the strength of intact veined rock," Rock Mechanics and Rock Engineering, vol. 48, no. 5, pp. 1897-1912, 2015.

[21] N. Bahrani, B. Valley, and P. K. Kaiser, "Numerical simulation of drilling-induced core damage and its influence on mechanical properties of rocks under unconfined condition," 
International Journal of Rock Mechanics and Mining Sciences, vol. 80, pp. 40-50, 2015.

[22] W. Yuan, W. Wang, X. B. Su et al., "Numerical study of the impact mechanism of decoupling charge on blasting-enhanced permeability in low-permeability sandstones," International Journal of Rock Mechanics and Mining Sciences, vol. 106, pp. 300-310, 2018.

[23] Q. Ma, Y. L. Tan, X. S. Liu, Z. H. Zhao, and D. Y. Fan, "Mechanical and energy characteristics of coal-rock composite sample with different height ratios: a numerical study based on particle flow code," Environmental Earth Sciences, vol. 80, no. 8, p. 309, 2021.

[24] Itasca Consulting Group, PFC. 5.0 Manual, Itasca Consulting Group, Minneapolis, MN, USA, 2015.

[25] China Architecture \& Building Press, Numerical Simulation Technology and Application with Particle Flow Code (Pfc5.0), China Architecture and Building Press, Chong Shi, China, 2018.

[26] W. L. Gao, Z. H. Zhang, B. J. Li, and K. P. Li, "Study on numerical Simulation of geometric elements of blasting funnel based on PFC5.0," Shock and Vibration, vol. 2021, Article ID 8812964, 13 pages, 2021.

[27] C. Y. Liu, J. X. Yang, and B. Yu, "Rock-breaking mechanism and experimental analysis of confined blasting of borehole surrounding rock," International Journal of Mining Science and Technology, vol. 27, no. 5, pp. 795-801, 2017.

[28] X. T. Feng, Y. Yu, G. L. Feng, Y. X. Xiao, B. R. Chen, and Q. Jiang, "Fractal behaviour of the microseismic energy associated with immediate rockbursts in deep, hard rock tunnels," Tunnelling and Underground Space Technology, vol. 51, pp. 98-107, 2016.

[29] X. S. Liu, D. Y. Fan, Y. L. Tan et al., "New detecting method on the connecting fractured zone above the coal face and a case study," Rock Mechanics and Rock Engineering, vol. 54, no. 8, pp. $4379-4391,2021$.

[30] A. B. Jin, H. Sun, G. W. Ma, Y. T. Gao, S. C. Wu, and X. Q. Meng, "A study on the draw laws of caved ore and rock using the discrete element method," Computers and Geotechnics, vol. 80, pp. 59-70, 2016.

[31] J. Bi and X. P. Zhou, "Numerical simulation of kinetic friction in the fracture process of rocks in the framework of general particle dynamics," Computers and Geotechnics, vol. 83, pp. 1-15, 2017.

[32] J. A. Wang, L. Yang, F. Li, and C. Wang, "Force chains in top coal caving mining," International Journal of Rock Mechanics and Mining Sciences, vol. 127, Article ID 104218, 2020.

[33] N. P. Kruyt, "On weak and strong contact force networks in granular materials," International Journal of Solids and Structures, vol. 92-93, pp. 135-140, 2016. 